\title{
Interactions between favipiravir and a BNC cage towards drug delivery applications
}

\author{
Azar Asgari Pari ${ }^{1} \cdot$ Mohammad Yousefi ${ }^{2}$ (D)
}

Received: 5 August 2021 / Accepted: 1 September 2021 / Published online: 8 September 2021

(c) The Author(s), under exclusive licence to Springer Science+Business Media, LLC, part of Springer Nature 2021

\begin{abstract}
Electronic structure analysis of bimolecular formation of favipiravir (Fav) and a representative model of boron-nitrogen-carbon (BNC) cage was performed in this work for providing more insightful information regarding the drug delivery purposes by the importance of Fav drug for medication of COVID-19. To achieve the purpose of this work, density functional theory (DFT) calculations were carried out to obtain the stabilized structures and corresponding molecular and atomic scale descriptors. Six models of BNC-Fav complexes were obtained reading the participation of different atomic positions of Fav to interactions with the BNC cage surface. The results yielded BNC-Fav2 at the highest strength and BNC-Fav4 at the lowest strength of bimolecular formations. Molecular orbital-related features and atomic scale quadrupole coping constants all revealed that BNC-Fav2 complex could be proposed for employing in drug delivery process by managing the loaded Fav contribution to future interactions.
\end{abstract}

Keywords COVID-19 $\cdot$ Favipiravir $\cdot$ BNC cage $\cdot$ Nanostructure $\cdot$ Molecular interactions $\cdot$ DFT

\section{Introduction}

By the end of year 2019, the world was shocked by an attack of a wide-spreading type of coronavirus causing the coronavirus disease (COVID-19) with harmful impacts on human health system and life worldwide [1-3]. To this date, the problem of treating COVID-19 has not been solved yet and the deathful impacts are still seen for the people [4-6]. Regarding the importance of this issue, considerable efforts of researchers have been dedicated to find a way of prevention or treatment of COVID-19, but no certain solution has been found up to now [7-9]. Therefore, performing further research works on the topic of COVID-19 is still a must to be done [10-12]. Among the investigated examinations, favipiravir (Fav) has been seen a useful drug for medication of coronavirus-infected patients

Mohammad Yousefi

myousefi50@hotmail.com

1 Department of Chemistry, Yadegar-e Imam Khomeini (RAH) Shahr-e-Rey Branch, Islamic Azad University, Tehran, Iran

2 Department of Chemistry, Faculty of Pharmaceutical Chemistry, Tehran Medical Sciences, Islamic Azad University, Tehran, Iran to save their life or increase health level [13-15]. To this point, several attempts have been done to show advantages and disadvantages of medication of patients by Fav, in which there is still no certain achievement about its prescription [16-18]. Therefore, several researchers have tried to focus on improving the features of Fav for more efficient prescription and medication [19-21]. Employing carriers has been also proposed for targeted delivery of Fav to the infected tissues, in which such carriers should be carefully investigated for appropriate application [22-24]. Nanostructures have been always expected to play import role of carriers in drug delivery processes, in which they have been also assumed to work for delivery of Fav [25-27]. However, the works are still under improvement regarding achievements of researchers of various fields of study [28-30]. Several attempts have been always dedicated to develop procedures of identifications of components living systems [31, 32]. Among the methods of dealing with such problems, computer-based methods based on electronic structure concepts could help to provide insightful information for going forward to reach a proposed solution for such a complicated problem [33-35]. Indeed, molecular and atomic scale descriptors of matters could be obtained by computer-based works to investigate the characteristic features of matters at the smallest scales [36-38]. 
In this work, the electronic structure features of Fav were analyzed in combination with a representative nanostructure as an estimated carrier of Fav for drug delivery purposes. To approach this goal, quantum calculations were performed to stabilize each of the singular and bimolecular models to show the impacts of nanostructure on the characteristic features of loaded Fav. The representative nanostructure was a fullerene-like cage structure with boron, nitrogen, and carbon atomic counterparts with the stoichiometry of $\mathrm{C}_{8} \mathrm{~B}_{6} \mathrm{~N}_{6}$ called a BNC cage. It is noted that cage structures have been seen as interesting for investigations by earlier works [39-41]. Singular models of Fav and BNC are shown in Figs. 1 and 2. After obtaining optimized singular structures, their combination for BNC-Fav bimolecular models (Fig. 3) was investigated by performing additional optimization calculations and evaluating the corresponding electronic structure features. The obtained molecular and atomic scale descriptors are summarized in Tables 1 and 2 and the visual representations are exhibited in Figs. 1-4 to approach the goal of this work.

\section{Materials and methods}

The major materials of this work were Fav $\left(\mathrm{C}_{5} \mathrm{H}_{4} \mathrm{FN}_{3} \mathrm{O}_{2}\right)$ and $\mathrm{BNC}\left(\mathrm{C}_{8} \mathrm{~B}_{6} \mathrm{~N}_{6}\right)$ models, in which their bimolecular formations were investigated by generating further materials (Figs. 1-3). First, the singular models were optimized to obtain the stabilized strictures. Next, the already singular models were combined with each other through various positioning faces resulting in six bimolecular models, from BNC-Fav1 up to BNC-Fav6 as shown in Fig. 3. Hence, the stoichiometry of bimolecular models was identical but

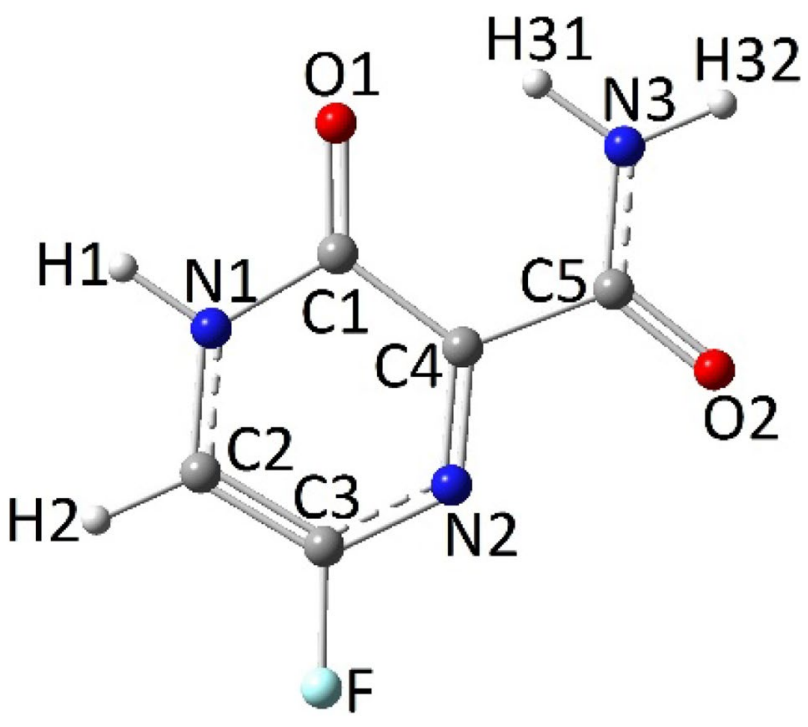

Fig. 1 Favipiravir (Fav)

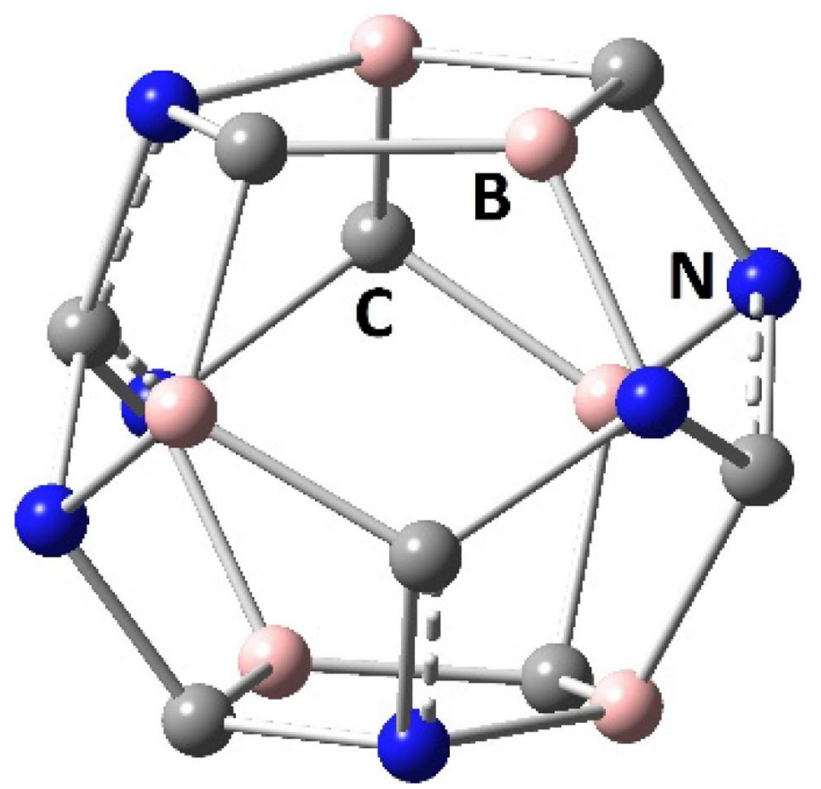

Fig. 2 BNC cage

their relaxing configurations were different. To stabile the strictures, optimization calculations were performed using the WB97XD/6-31+G* level of density functional theory (DFT) as implemented in the Gaussian program [42]. By doing such calculations, the models were prepared for running further analyses of electronic structure features. To this aim, values of total energy $\left(E_{\text {tot }}\right)$, interaction energy $\left(E_{\text {int }}\right)$, and the basis set superposition error (BSSE) were evaluated for the model systems. Furthermore, molecular orbital features including energies of the highest occupied and the lowest unoccupied molecular orbitals (HOMO and LUMO), energy gap $\left(E_{\text {gap }}\right)$, and chemical hardness and softness $(\eta$ and $\sigma$ ) were evaluated for the optimized models. Additionally, values of dipole moment $\left(D_{\mathrm{m}}\right)$ were evaluated for the investigated models to show electric charge distribution orientation. All the obtained descriptors for the optimized models are summarized in Table 1 . In addition to quantitative descriptors, qualitative representations of optimized models are exhibited in Figs. 1-3, and distribution patterns for HOMO and LUMO are exhibited in Fig. 4 in addition to the visualized electrostatic potential (ESP) surfaces. As a result of performing such computations, the required features of molecular scale were provided for analyzing electronic structure features of the models. To analyze atomic features of Fav, values of quadrupole coupling constants $\left(C_{\mathrm{q}}\right)$ were evaluated for hydrogen, nitrogen, and oxygen atoms of Fav to show the impacts of bimolecular formation with BNC cage on the original features of Fav at the atomic scale. The obtained values of $C_{\mathrm{q}}$ are summarized in Table 2 . The values of $C_{\mathrm{q}}$ could show the localized electron density at the atomic sites with sensitivity to effects of any perturbations to the 
BNC-Fav1 >

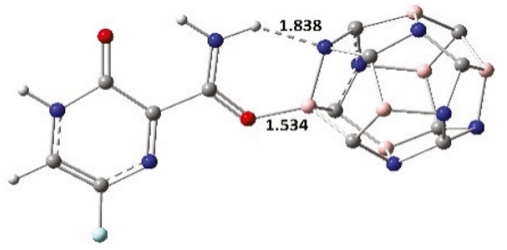

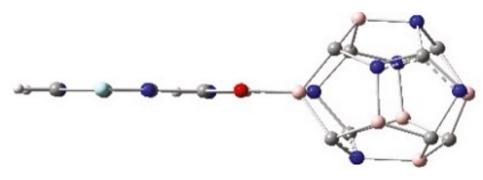

BNC-Fav2 >
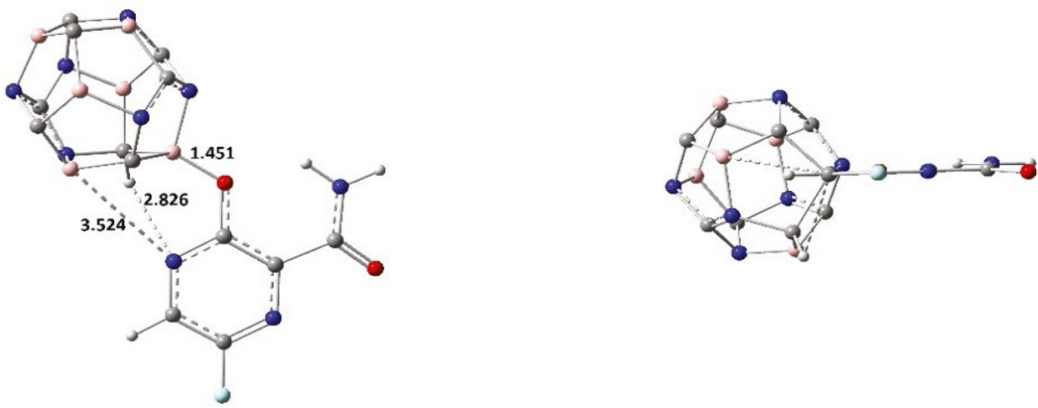

BNC-Fav3 >
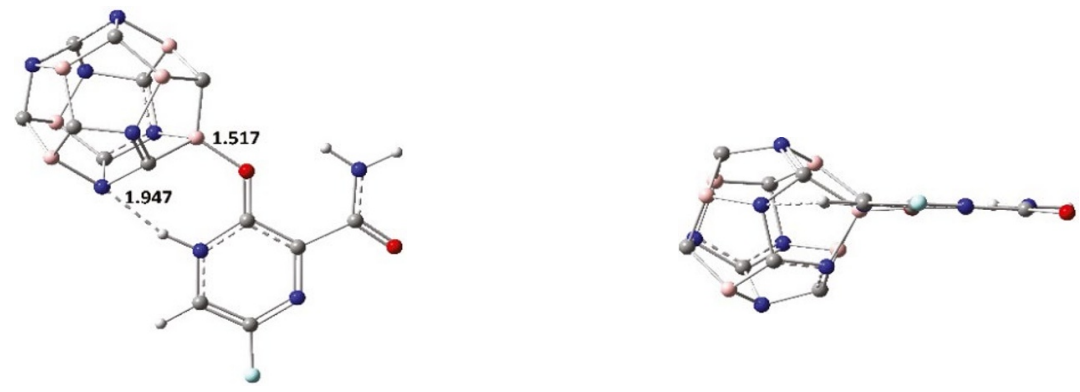

BNC-Fav4 >
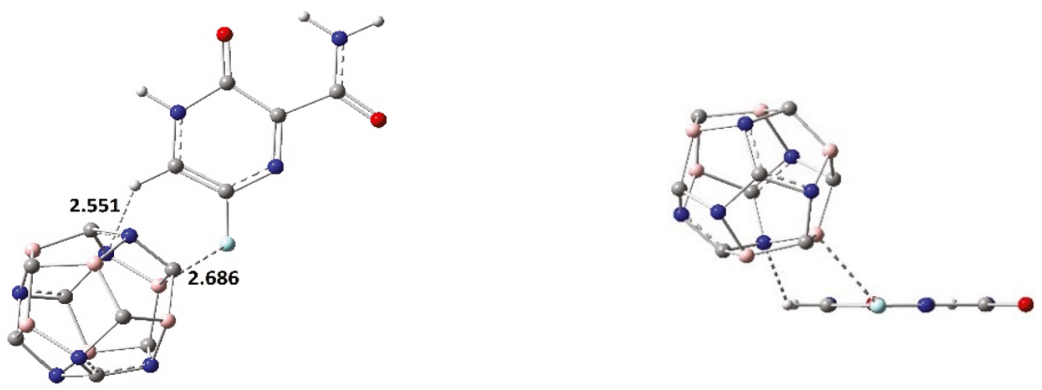

BNC-Fav5 >
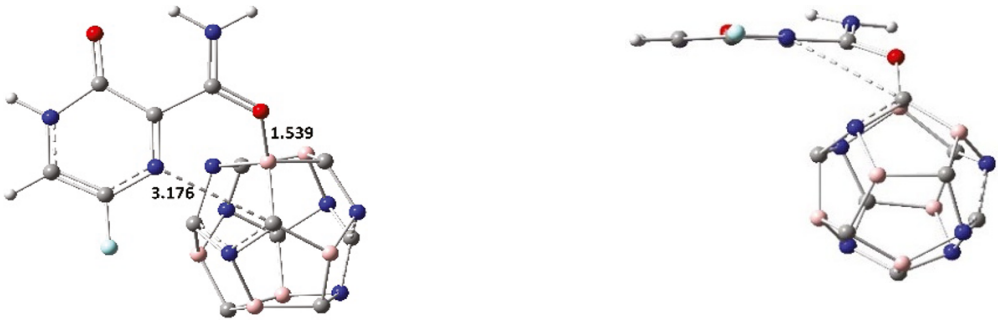

BNC-Fav6 >
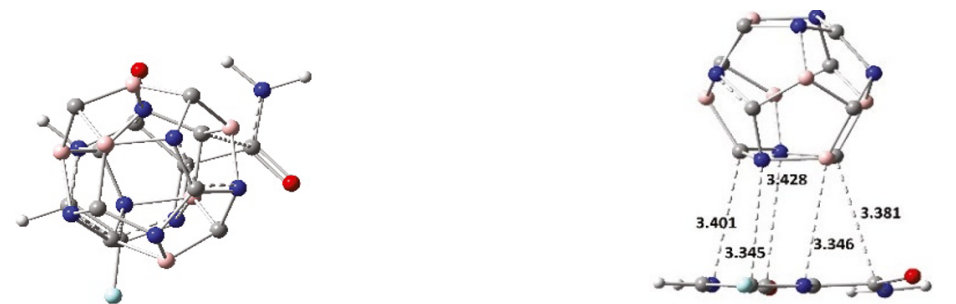

Fig. 3 Different views of the bimolecular complex models. Intermolecular distances $(\AA)$ are shown 
Table 1 The obtained descriptors for the optimized models*

\begin{tabular}{lllllllllr}
\hline Model & $\begin{array}{l}\boldsymbol{E}_{\text {tot }} \\
\mathrm{eV}\end{array}$ & $\begin{array}{l}\boldsymbol{E}_{\text {int }} \\
\mathrm{eV}\end{array}$ & $\begin{array}{l}\text { BSSE } \\
\mathrm{eV}\end{array}$ & $\begin{array}{l}\text { HOMO } \\
\mathrm{eV}\end{array}$ & $\begin{array}{l}\text { LUMO } \\
\mathrm{eV}\end{array}$ & $\begin{array}{l}\boldsymbol{E}_{\text {gap }} \\
\mathrm{eV}\end{array}$ & $\begin{array}{l}\boldsymbol{\eta} \\
\mathrm{eV}\end{array}$ & $\begin{array}{l}\boldsymbol{\sigma} \\
\mathrm{eV}^{-1}\end{array}$ & $\begin{array}{l}\boldsymbol{D}_{\mathrm{m}} \\
\text { Debye }\end{array}$ \\
\hline Fav $>$ & -16526.320 & $\mathrm{n} / \mathrm{a}$ & $\mathrm{n} / \mathrm{a}$ & -9.064 & -1.188 & 7.876 & 3.938 & 0.254 & 6.263 \\
BNC $>$ & -21278.094 & $\mathrm{n} / \mathrm{a}$ & $\mathrm{n} / \mathrm{a}$ & -7.628 & -2.187 & 5.440 & 2.720 & 0.368 & 1.984 \\
BNC-Fav1 > & -37805.830 & -1.416 & 0.087 & -6.363 & -2.347 & 4.016 & 2.008 & 0.498 & 16.113 \\
BNC-Fav2 $>$ & -37807.883 & -3.469 & 0.035 & -7.954 & -2.375 & 5.579 & 2.790 & 0.358 & 7.581 \\
BNC-Fav3 $>$ & -37805.847 & -1.433 & 0.009 & -7.088 & -2.398 & 4.690 & 2.345 & 0.426 & 5.235 \\
BNC-Fav4 > & -37804.588 & -0.174 & 0.036 & -7.728 & -2.293 & 5.436 & 2.718 & 0.368 & 6.626 \\
BNC-Fav5 > & -37805.595 & -1.180 & 0.014 & -6.297 & -2.213 & 4.083 & 2.042 & 0.490 & 12.906 \\
BNC-Fav6 > & -37804.753 & -0.339 & 0.071 & -7.602 & -2.175 & 5.427 & 2.713 & 0.369 & 5.768 \\
\hline
\end{tabular}

*See Figs. 1-3 for visual descriptions of the models atomic sites especially for the interacting systems [43-45]. It is important to mention that several parameters are required for analyzing electronic structure features of matters, in which computer-based works could help to provide reliable results for approaching such goal [46-48].

\section{Results and discussion}

The major goal of this work was to perform an electronic structure analysis of the bimolecular formation of favipiravir (Fav) and a representative BNC cage for drug delivery purposes through DFT calculations. To approach this goal, singular and bimolecular models were optimized to reach stabilized structures as presented in Figs. 1-3. Although there were only two stating singular models for participating in bimolecular formations, six bimolecular models of BNC-Fav were obtained based on various relaxing configurations of molecular counterparts towards each other. As a consequence, the optimized models were prepared for evaluating molecular and atomic scale descriptors (Tables 1 and 2), and for visualized HOMO and LUMO distribution patterns and ESP surfaces (Fig. 4). To this point, all required quantitative and qualitative descriptors were evaluated for the investigated models to show the impacts of such bimolecular formation on the original features of Fav to propose a possible carrier for more efficient drug delivery purposes.
It is important to mention that carbon nanostructures have been always expected to work properly for pharmaceutical applications in living systems [49-51]. However, their hydrophobicity raised an idea of developing impure carbon nanostructures by substituting other atoms instead of some or all available carbon atoms to reduce hydrophobicity feature [52-54]. In this case, combinations of boron and nitrogen (BN) atoms have produced excellent single-standing boron nitride nanostructures of even heteroatomic $\mathrm{BN}$-substituted carbon nanostructures [55-57]. As a consequence, the investigated cage particle of this work was a combination of boron, nitrogen, and carbon atoms to construct the BNC cage (Fig. 2) for playing a possible carrier role of Fav (Fig. 1). Indeed, heterosurface of the BNC cage could make possible the interacting molecules to contribute to different types of interactions in comparison with the identical surface of a pure carbon cage structure. Such benefit led to the formation of six bimolecular BNC-Fav models (Fig. 3) to analyze electronic structure features to show impacts of the existence of the BNC cage on the original features of Fav.

The obtained bimolecular models of BNC-Fav complexes indicated the variation of complex formation regarding the relaxed configurations of molecular counterparts towards each other. In this case, those atomic sites of Fav counterpart with a potency of contributing to interactions participated in interactions with the heterosurface of the BNC cage. To make visual such note about such interacting bimolecular
Table 2 The obtained quadrupole coupling constants $\left(\mathrm{C}_{\mathrm{q}} \mathrm{MHz}\right)$ for the Fav of optimized models*

\begin{tabular}{llllllllll}
\hline Model & H1 & H2 & H31 & H32 & N1 & N2 & N3 & O1 & O2 \\
\hline Fav $>$ & 0.249 & 0.202 & 0.236 & 0.266 & 2.632 & 5.348 & 3.673 & 8.143 & 9.054 \\
BNC-Fav1 > & 0.246 & 0.200 & 0.226 & 0.185 & 2.448 & 5.435 & 2.001 & 8.108 & 6.048 \\
BNC-Fav2 > & 0.202 & 0.199 & 0.250 & 0.266 & 4.480 & 5.082 & 3.870 & 8.834 & 9.179 \\
BNC-Fav3 > & 0.183 & 0.199 & 0.249 & 0.262 & 1.434 & 5.449 & 3.753 & 8.691 & 9.135 \\
BNC-Fav4 > & 0.249 & 0.198 & 0.236 & 0.265 & 2.606 & 5.342 & 3.674 & 8.164 & 9.050 \\
BNC-Fav5 > & 0.247 & 0.201 & 0.222 & 0.255 & 2.506 & 5.304 & 2.772 & 7.987 & 8.156 \\
BNC-Fav6 > & 0.249 & 0.202 & 0.235 & 0.265 & 2.570 & 5.292 & 3.623 & 8.168 & 9.038 \\
\hline
\end{tabular}

*See Figs. 1 and 3 for visual descriptions of the models 
Fav >
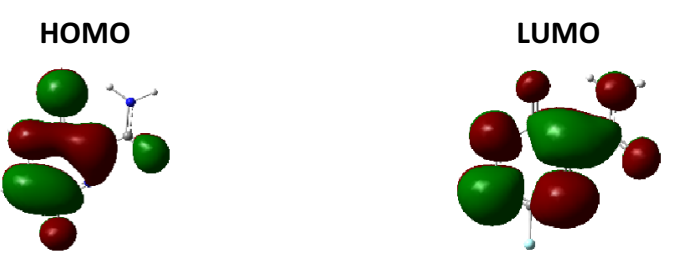

ESP

BNC >
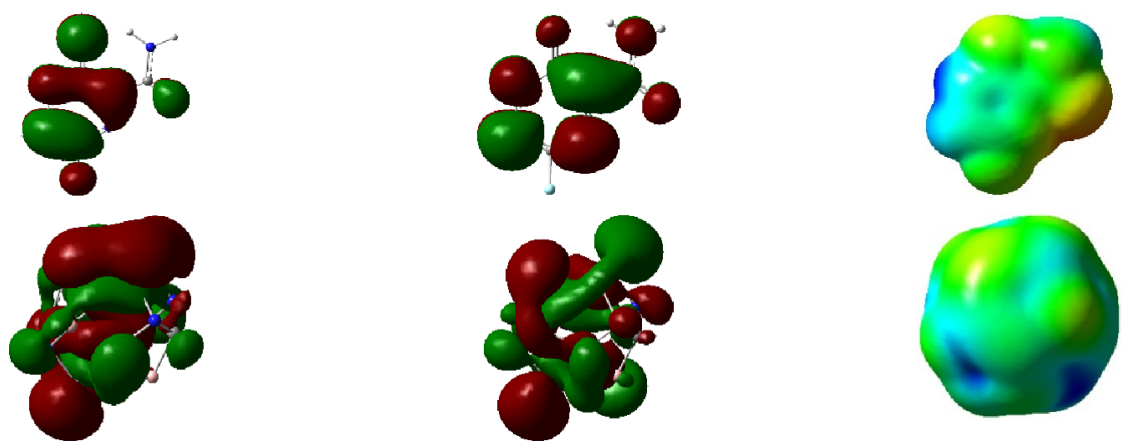

BNC $>$

BNC-Fav1 >
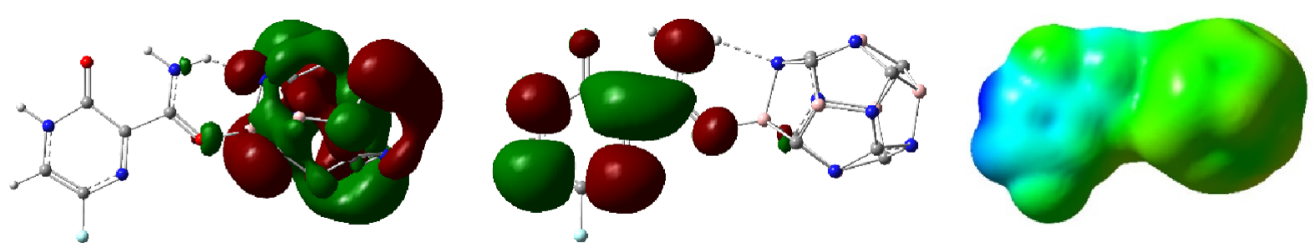

BNC-Fav2 >
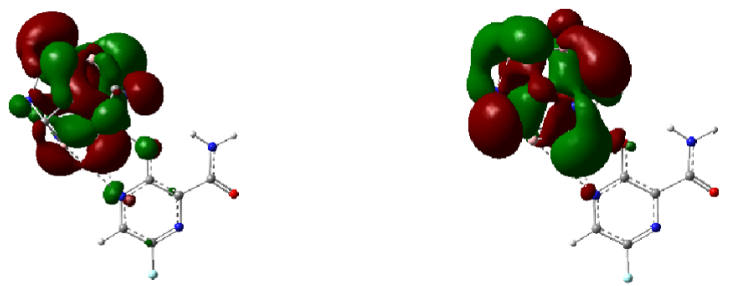

BNC-Fav3 >
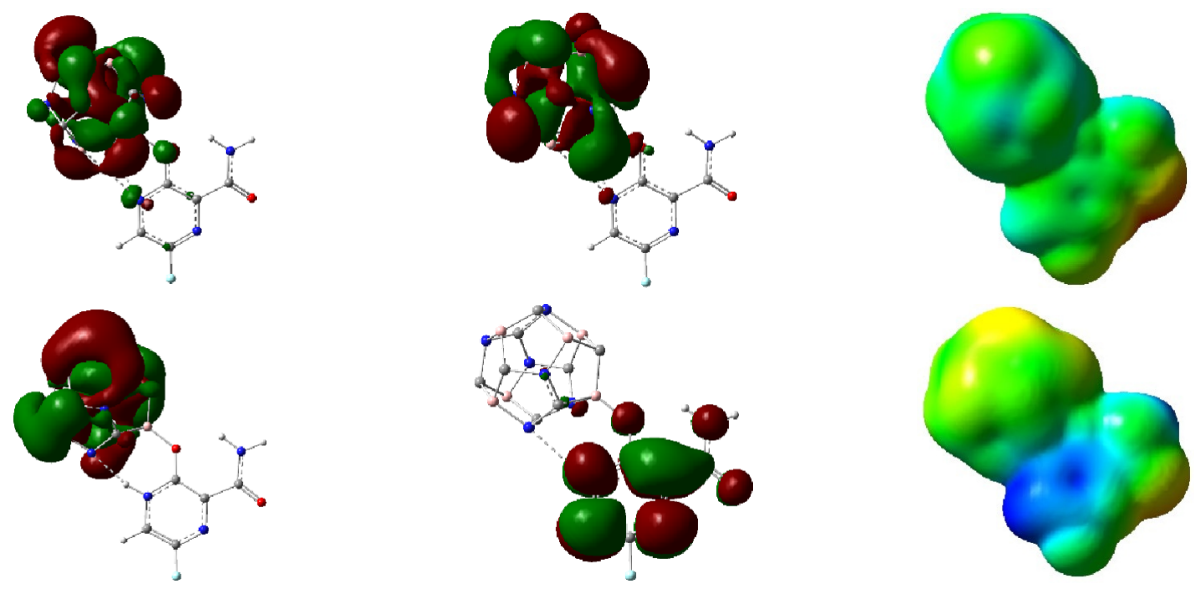

BNC-Fav4 >
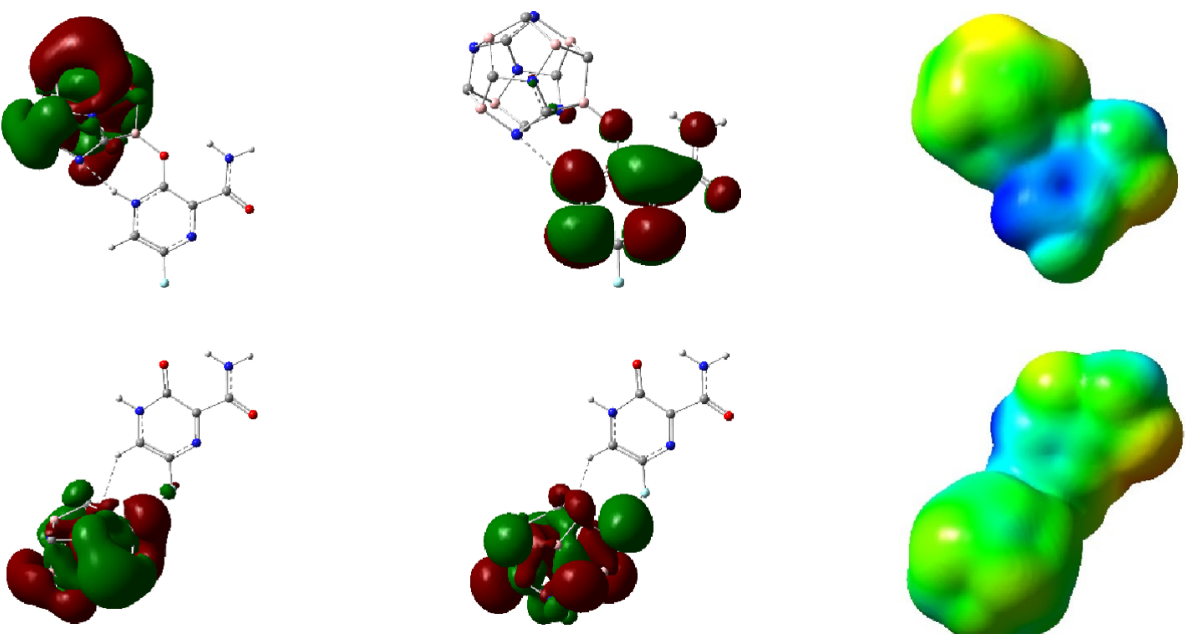

\section{BNC-Fav4 >}

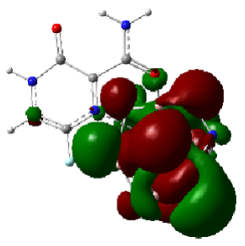

BNC-Fav6 >
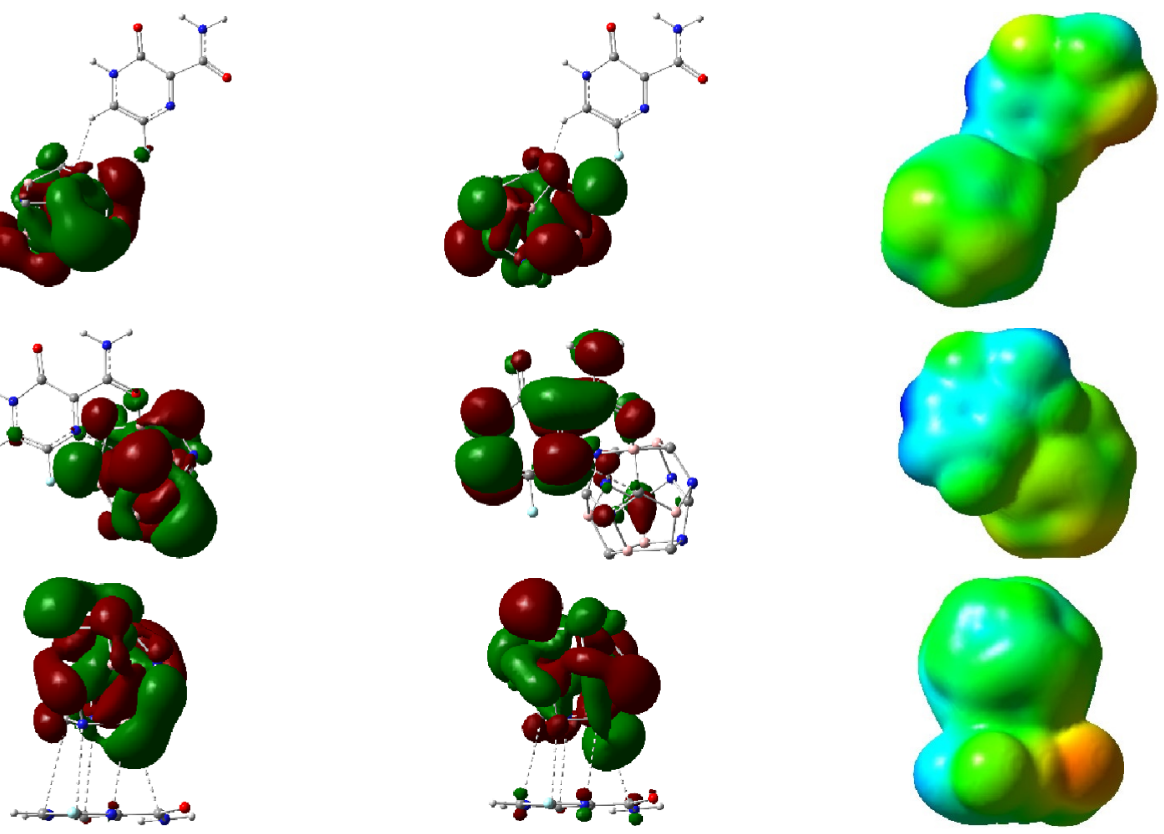

BNC-Fav5 >
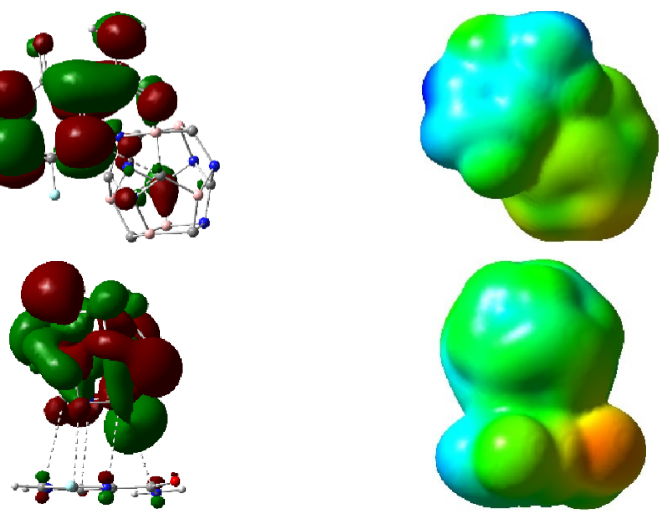

Fig. 4 HOMO-LUMO distribution patterns and ESP surfaces 
formations, analyzing the visualized ESP surfaces of Fig. 4 could help to reach brighter points. As shown by different colors for ESP surfaces of each of singular Fav and BNC, various possibilities of interactions could be expected for the formation of such bimolecular complex systems. Blue color stands for positive, green color stands for neutral, and yellow and red colors stand for negative electrostatic parts of a molecule. As a result, the models showed colorful ESP for making them possible for participating in various interacting configurations. To this point, six models were obtained for BNC-Fav complex systems, in which their strengths are more or less different from each other. As listed in Table 1, BNC-Fav2 and BNC-Fav4 were the strongest and the weakest models for the investigated complexes, respectively. Both values of $E_{\text {tot }}$ and $E_{\text {int }}$ approved such observation for the highest and lowest strength of BNC-Fav bimolecular models. To follow the structures visually, the corresponding panels of Fig. 3 showed the formation of a covalent bond for $\mathrm{O} 1$ atom of Fav and $\mathrm{B}$ atom of the BNC cage in addition to the formation of other interactions between two counterparts of the BNC-Fav2 complex. However, there were not any appropriate atomic positions for making strong interactions between the molecular counterparts in the BNC-Fav4 model resulting in the weakest complex systems for the BNC-Fav models. There might be a question about the importance of investigating formations of such weak complexes, in which it should be mentioned that several parameters could favor the formation of interacting systems even the formation of weak complex systems. Comparing to experiments, the efficiency of reaction products could be managed by conducting the molecules to be localized in the most appropriate configurations for interactions. This is indeed an advantage of performing such computer-based works to show the possibility of all complex formations for providing more insightful results for the investigated systems. For the investigated complexes, the order of strength could be ranked regarding the obtained values of both $E_{\text {tot }}$ and $E_{\text {int }}$ parameters: BNCFav2 > BNC-Fav3 > BNC-Fav1 > BNC-Fav5 > BNC-Fav6 $>$ BNC-Fav4. The obtained values of BSSE were not meaningful to make significant changes to the obtained energies of interacting complex systems. Besides the participation of atomic sites of Fav in interactions with atomic sites of the BNC cage, whole structures of two molecules were also interacting together to make the BNC-Fav6 complex with somehow parallel positioning of $\mathrm{Fav}$ at the partial surface of the BNC cage.

One important point about obtaining such different complexes is the existence of the heterosurface of the BNC cage in comparison with the homosurface of the pure carbon cage. The presence of impurities could increase the number of configurations suitable for the interactions [58-60]. Further analysis of the models based on HOMO and LUMO features could indicate variations of such molecular orbital energy levels for the complex models in comparison with singular forms of both of Fav and BNC cage. It is known that HOMO level could imply for the tendency of the molecule for electron donating whereas LUMO level could imply for such tendency for electron accepting. By such definitions, the investigated models showed different tendencies of participating in such electron-transferring processes. In addition to such levels, energy distance between HOMO and LUMO, assigned by $E_{\text {gap }}$, is also another important feature for showing the reactivity modes of molecular systems. Additionally, each of the hardness and softness ( $\eta$ and $\sigma$ ) parameters could lead to the favorability of molecules for participating in electron-transferring processes. Such variations of electric features were also observed by the obtained values of $D_{\mathrm{m}}$. Examining the visual representations of HOMO and LUMO in Fig. 4 could show a very important achievement for the investigated model systems. As expected for nanostructures to work as proper carriers for drug delivery processes, it was seen that both HOMO and LUMO patterns were moved to the cage part in the strongest BNC-Fav2 model. This is indeed showing a dominant role of the employed BNC cage for managing the participation of loaded Fav in future interactions. Localization of HOMO and LUMO at the molecules could show the tendency of molecular parts for contributing to further interactions, in which such tendency was eliminated for Fav part in the BNC-Fav2 complex. As a result, it could be suggested that the formation of BNC-Fav2 complex might somehow reduce the unwanted side effects of medication of Fav for the patients, but further investigations are still required. As a consequence, BNC-Fav2 could be proposed for the best model of complex formation of Fav and BNC cage for further analyzing of applications developments.

As mentioned about the importance of atomic positions of Fav for contributing to interactions with the BNC cage, the values of quadrupole coupling constants $\left(C_{\mathrm{q}}\right)$ were evaluated for $\mathrm{H}, \mathrm{N}$, and $\mathrm{O}$ atoms of Fav counterpart to show the impacts of complex formation at the smallest atomic scale features [61-63]. Different atomic types actually imply for different electronic environments, which are the most important factors for atoms to determine their participation in future interactions. The magnitude of such electronic density localization at the atomic site could be determined by values of $C_{\mathrm{q}}$, in which such magnitudes could be analyzed before and after the participation of atoms in interactions to show the perturbating impacts exactly. As shown in Fig. 1, the atoms were numbered for being detectable in the atomic analysis regarding the obtained values of $C_{\mathrm{q}}$ as listed in Table 2. A quick look at the obtained results could mean that the electronic sites of all atoms of Fav detected a more or less significant impact of complex formation in BNC-Fav models. As detected by energy features, BNC-Fav2 was the strongest complex, in which analyzing values of $C_{\mathrm{q}}$ for each of $\mathrm{H} 1, \mathrm{~N} 1$, and $\mathrm{O} 1$ 
of singular and complex models show significant effects of interactions on the features of these atoms. Moreover, slight changes in electronic features were also detected for the rest of the atoms of Fav in the BNC-Fav2 model. Interestingly, the changes of atomic features of Fav from singular to complex model were almost negligible for the BNC-Fav4 model, as indicated earlier as the weakest complex formation for the investigated models. For other models, more or less significant impacts were observed for the atomic features by comparing the singular and complex models. Indeed, this is important how to decide for future functions of drug compounds in the drug delivery processes to employ the appropriate carrier for them. As a consequence, the atomic features of Fav were almost completely managed by the BNC cage assigning a carrier with significant impacts on the loaded drug.

\section{Conclusion}

The major goal of this work was to perform an electronic structure analysis of the bimolecular formation of Fav and BNC cage for providing more insightful information regarding drug delivery purposes. To this aim, the obtained results indicated that such an idea of BNC-Fav bimolecular formation was achievable based on the obtained values of energies for whole structures and interacting modes. To this point, the obtained complex formations were ordered regarding their strength, in which BNC-Fav2 was found to be the strongest one, whereas BNC-Fav4 was found to be the weakest one. Such strengths were obtained by the importance of atomic types and positions of Fav counterpart in addition to the appropriate heteroatomic surface of the BNC cage. As a consequence, complexes with different strengths were obtained for the investigated models. Further analysis of molecular orbital features also indicated different tendencies for participating in interactions for the complex models even different from those singular counterparts. Atomic scale $C_{\mathrm{q}}$ parameters showed the exact roles of atomic sites for the investigated models, in which their values were more or less significant for atoms of the whole structure. Interestingly, the most and the least significant effects were observed for atoms of BNC-Fav2 and BNC-Fav4, respectively, as were assigned for the highest and the lowest strength of the investigated BNC-Fav complexes. As a consequence, the BNC cage was seen useful for employing in the drug delivery process of loaded Fav for further investigations.

Author contribution Conceptualization: [Azar asgari pari], [Mohammad Yousefi]. Methodology: [Azar asgari pari]. Formal analysis and investigation: [Azar asgari pari]. Writing - original draft preparation: Azar asgari pari. Writing - review and editing: [Mohammad Yousefi]. Supervision: [Mohammad Yousefi].

\section{Declarations}

Conflict of interest The authors declare no competing interests.

\section{References}

1. Velavan TP, Meyer CG (2020) The COVID-19 epidemic. Trop Med Int Health 25:278-280

2. Arshizadeh S, Gorgani SH, Taheri P, Givgol M, Shahrokhi S, Abdalisousan A (2021) The impact of COVID-19 on oil supply in the short term. Adv J Sci Eng 2:120-135

3. Ozkendir OM, Askar M, Kocer NE (2020) Influence of the epidemic COVID-19: an outlook on health, business and scientific studies. Lab-in-Silico 1:26-30

4. He H, Harris L (2020) The impact of COVID-19 pandemic on corporate social responsibility and marketing philosophy. J Bus Res 116:176-182

5. Harismah K, Mirzaei M (2020) COVID-19: a serious warning for emergency health innovation. Adv J Sci Eng 1:32-33

6. Cotofan M, De Neve JE, Golin M, Kaats M, Ward G (2021) Work and well-being during COVID-19: impact, inequalities, resilience, and the future of work. World Happiness Report 2021 153-190

7. Goodarzi P, Mahdavi F, Mirzaei R, Hasanvand H, Sholeh M, Zamani F, Sohrabi M, Tabibzadeh A, Jeda AS, Niya MH, Keyvani H (2020) Coronavirus disease 2019 (COVID-19): Immunological approaches and emerging pharmacologic treatments. Int Immunopharmacol 106885

8. Mirzaei M, Harismah K, Da'i M, Salarrezaei E, Roshandel Z (2020) Screening efficacy of available HIV protease inhibitors on COVID-19 protease. J Mil Med 22:100-107

9. Marwitz KK (2021) The pharmacist's active role in combating COVID-19 medication misinformation. J Am Pharm Assoc 61:71-74

10. Nalbandian A, Sehgal K, Gupta A, Madhavan MV, McGroder C, Stevens JS, Cook JR, Nordvig AS, Shalev D, Sehrawat TS, Ahluwalia N (2021) Post-acute COVID-19 syndrome. Nat Med 27:601-615

11. Yang Y, Liu J, Zhou X (2021) A CRISPR-based and post-amplification coupled SARS-CoV-2 detection with a portable evanescent wave biosensor. Biosens Bioelectron 190:113418

12. Paredes MR, Apaolaza V, Fernandez-Robin C, Hartmann P, Yañez-Martinez D (2021) The impact of the COVID-19 pandemic on subjective mental well-being: the interplay of perceived threat, future anxiety and resilience. Pers Individ Differ 170:110455

13. Vora A, Tiwaskar M (2020) Favipiravir. J Assoc Phys India 68:91-92

14. Takahashi H, Iwasaki Y, Watanabe T, Ichinose N, Okada Y, Oiwa A, Kobayashi T, Moriya M, Oda T (2020) Case studies of SARS$\mathrm{CoV}-2$ treated with favipiravir among patients in critical or severe condition. Int J Infect Dis 100:283-285

15. Naydenova K, Muir KW, Wu LF, Zhang Z, Coscia F, Peet MJ, Castro-Hartmann P, Qian P, Sader K, Dent K, Kimanius D (2021) Structure of the SARS-CoV-2 RNA-dependent RNA polymerase in the presence of favipiravir-RTP. Proc Natl Acad Sci U S A 118:2021946118

16. Çeviker SA, ŞENER A, Yüksel C, Önder T, Anıl AK, Vurucu S, Kayta SB (2020) Angioedema and acute urticaria in a patient 
with COVID 19 pneumonia: Favipiravir side effect or COVID-19 cutaneous manifestation. J Emerg Med Case Rep 12:65-67

17. Dabbous HM, Abd-Elsalam S, El-Sayed MH, Sherief AF, Ebeid FF, Abd El Ghafar MS, Soliman S, Elbahnasawy M, Badawi R, Tageldin MA (2021) Efficacy of favipiravir in COVID-19 treatment: a multi-center randomized study. Arch Virol 166:949-954

18. Harismah K, Mirzaei M (2020) Favipiravir: structural analysis and activity against COVID-19. Adv J Chem B 2:55-60

19. Manabe T, Kambayashi D, Akatsu H, Kudo K (2021) Favipiravir for the treatment of patients with COVID-19: a systematic review and meta-analysis. BMC Infect Dis 21:1-3

20. Lou Y, Liu L, Yao H, Hu X, Su J, Xu K, Luo R, Yang X, He L, Lu X, Zhao Q (2021) Clinical outcomes and plasma concentrations of baloxavir marboxil and favipiravir in COVID-19 patients: an exploratory randomized, controlled trial. Eur J Pharm Sci 157:105631

21. Dabbous HM, El-Sayed MH, El Assal G, Elghazaly H, Ebeid FF, Sherief AF, Elgaafary M, Fawzy E, Hassany SM, Riad AR, TagelDin MA (2021) Safety and efficacy of favipiravir versus hydroxychloroquine in management of COVID-19: a randomised controlled trial. Sci Rep 11:1-7

22. Yuksel N, Köse A, Fellah MF (2021) The supramolecularly complexes of calix [4] arene derivatives toward favipiravir antiviral drug (used to treatment of COVID-19): a DFT study on the geometry optimization, electronic structure and infrared spectroscopy of adsorption and sensing. J Incl Phenom Macrocycl Chem 5:1-3

23. Rad AS, Ardjmand M, Esfahani MR, Khodashenas B (2021) DFT calculations towards the geometry optimization, electronic structure, infrared spectroscopy and UV-vis analyses of Favipiravir adsorption on the first-row transition metals doped fullerenes; a new strategy for COVID-19 therapy. Spectrochim Acta A Mol Biomol Spectrosc 247:119082

24. Driouich JS, Cochin M, Lingas G, Moureau G, Touret F, Petit PR, Piorkowski G, Barthélémy K, Laprie C, Coutard B, Guedj J (2021) Favipiravir antiviral efficacy against SARS-CoV-2 in a hamster model. Nat Commun 12:1735

25. Aramideh M, Mirzaei M, Khodarahmi G, Gülseren O (2017) DFT studies of graphene-functionalised derivatives of capecitabine. $\mathrm{Z}$ Naturforsch A 72:1131-1138

26. Deng Y, Zhang X, Shen H, He Q, Wu Z, Liao W, Yuan M (2020) Application of the nano-drug delivery system in treatment of cardiovascular diseases. Front Bioeng Biotechnol 7:489

27. Mirzaei M, Hadipour N, Gulseren O (2021) DNA codon recognition by a cubane wire: in silico approach. Turk Comput Theor Chem 5:13-19

28. Zandi H, Harismah K (2021) Density functional theory analyses of non-covalent complex formation of 6-thioguanine and coronene. Lab-in-Silico 2:57-62

29. Kakaei A, Mirzaei M (2021) Cyclophosphamide@CNT: in silico exploration of nano drug delivery system. Lab-in-Silico 2:9-14

30. Fang X, Cao J, Shen A (2020) Advances in anti-breast cancer drugs and the application of nano-drug delivery systems in breast cancer therapy. J Drug Deliv Sci Technol 57:101662

31. Niu M, Lin Y, Zou Q (2021) sgRNACNN: identifying sgRNA on-target activity in four crops using ensembles of convolutional neural networks. Plant Mol Biol 105:483-495

32. Sun S, Xu L, Zou Q, Wang G (2021) BP4RNAseq: a babysitter package for retrospective and newly generated RNA-seq data analyses using both alignment-based and alignment-free quantification method. Bioinformatics 37:1319-1321

33. Yaghoobi R, Mirzaei M (2020) Computational analyses of cytidine and aza-cytidine molecular structures. Lab-in-Silico 1:21-26

34. Mirzaei M, Ravi S, Yousefi M (2012) Modifying a graphene layer by a thymine or a uracil nucleobase: DFT studies. Superlattices Microstruct 52:306-311
35. Harismah K, Mirzaei M, Moradi R (2018) DFT studies of single lithium adsorption on coronene. Z Naturforsch A 73:685-691

36. Zandi H, Harismah K (2021) Computer-based tools for structural characterizations and activity specifications of natural products: a quick review. Lab-in-Silico 2:50-54

37. Yaraghi A, Ozkendir OM, Mirzaei M (2015) DFT studies of 5-fluorouracil tautomers on a silicon graphene nanosheet. Superlattices Microstruct 85:784-788

38. Harismah K, Hajali N, Zandi H (2021) 6-Thioguanine bimolecular formation for dual chelation of iron: DFT study. Comput Theor Chem 1202:113308

39. Bamberg M, Bursch M, Hansen A, Brandl M, Sentis G, Kunze L, Bolte M, Lerner HW, Grimme S, Wagner M (2021) [Cl@ $\mathrm{Si20H20]-:} \mathrm{parent} \mathrm{siladodecahedrane} \mathrm{with} \mathrm{endohedral} \mathrm{chloride}$ ion. J Am Chem Soc 143:10865-10871

40. Ponce-Vargas M, Munoz-Castro A (2018) Stabilizing role of halide ions in endohedral [20] silafulleranes: insights from DFT calculations toward silicon nanocages. J Phys Chem C 122:12551-12558

41. Harismah K, Ozkendir OM, Mirzaei M (2020) Lithium adsorption at the C20 fullerene-like cage: DFT approach. Adv J Sci Eng 1:74-79

42. Frisch M, Trucks G, Schlegel H, Scuseria G, Robb M, Cheeseman J, Montgomery Jr J, Vreven, T, Kudin K, Burant J (2009) Gaussian 09 D.01 Program. Gaussian. Inc.: Wallingford, CT, USA

43. Piveteau L, Morad V, Kovalenko MV (2020) Solid-state NMR and NQR spectroscopy of lead-halide perovskite materials. J Am Chem Soc 142:19413-19437

44. Mirzaei M, Gulseren O, Hadipour N (2016) DFT explorations of quadrupole coupling constants for planar 5-fluorouracil pairs. Comput Theor Chem 1090:67-73

45. Mirzaei M, Yousefi M (2012) Computational studies of the purine-functionalized graphene sheets. Superlattices Microstruct 52:612-617

46. Rad AS, Aghaei SM, Pazoki H, Binaeian E, Mirzaei M (2018) Surface interaction of $\mathrm{H} 2 \mathrm{O}$ and $\mathrm{H} 2 \mathrm{~S}$ onto $\mathrm{Ca} 12 \mathrm{O} 12$ nanocluster: quantum-chemical analyses. Surf Interface Anal 50:411-419

47. Ozkendir OM, Cengiz E, Mirzaei M, Karahan IH, Ozdemir R, Klysubun W (2018) Electronic structure study of the bimetallic Cu1-xZnx alloy thin films. Mater Technol 33:193-197

48. Ozkendir OM (2020) Electronic structure study of Sn-substituted InP semiconductor. Adv J Sci Eng 1:7-11

49. Srivastava I, Misra SK, Bangru S, Boateng KA, Soares JA, Schwartz-Duval AS, Kalsotra A, Pan D (2020) Complementary oligonucleotide conjugated multicolor carbon dots for intracellular recognition of biological events. ACS Appl Mater Interfaces 12:16137-16149

50. Pandey H, Khare P, Singh S, Singh SP (2020) Carbon nanomaterials integrated molecularly imprinted polymers for biological sample analysis: a critical review. Mater Chem Phys 239:121966

51. Mirzaei M, Yousefi M (2013) Modified (n, 0) BN nanotubes ( $n=$ 3-10) by acetic acids: DFT studies. Superlattices Microstruct $55: 1-7$

52. Yousefi M, Afghahi SS, Amini MM, Torbati MB (2019) An investigation of structural and magnetic properties of $\mathrm{Ce}-\mathrm{Nd}$ doped strontium hexaferrite nanoparticles as a microwave absorbent. Mater Chem Phys 235:121722

53. Pagar T, Ghotekar S, Pansambal S, Pagar K, Oza R (2020) Biomimetic synthesis of $\mathrm{CuO}$ nanoparticle using Capparis decidua and their antibacterial activity. Adv J Sci Eng 1:133-137

54. Nasrollah A, Soleimanimehr H, Khazeni H (2021) Nafion-based ionic-polymer-metal composites: displacement rate analysis by changing electrode properties. Adv J Sci Eng 2:51-58

55. Faramarzi R, Falahati M, Mirzaei M (2020) Interactions of fluorouracil by CNT and BNNT: DFT analyses. Adv J Sci Eng 1:62-66 
56. Ariaei S (2021) DFT calculations of a cubic B4N4 cubane-like particle for CO gas adsorption. Adv J Sci Eng 2:93-98

57. Cao Y, Khan A, Mirzaei H, Khandoozi SR, Javan M, Lup AN, Norouzi A, Lemeski ET, Pishnamazi M, Soltani A, Albadarin AB (2021) Investigations of adsorption behavior and anti-cancer activity of curcumin on pure and platinum-functionalized B12N12 nanocages. J Mol Liq 334:116516

58. Sherafati M, Rad AS, Ardjmand M, Heydarinasab A, Peyravi M, Mirzaei M (2018) Beryllium oxide (BeO) nanotube provides excellent surface towards adenine adsorption: a dispersioncorrected DFT study in gas and water phases. Curr Appl Phys 18(9):1059-65

59. Ghotekar S, Pagar K, Pansambal S, Murthy HA, Oza R (2020) A review on eco-friendly synthesis of BiVO4 nanoparticle and its eclectic applications. Adv J Sci Eng 1:106-112

60. Yousefi M, Alimard P (2013) Synthesis of M-Nd doped Fe3O4 nanoparticles $(\mathrm{M}=\mathrm{Co}, \mathrm{Ce}, \mathrm{Cr}, \mathrm{Ni})$ with tunable magnetic properties. Bull Chem Soc Ethiop 27:49-56
61. Harismah K, Dhumad AM, Ibraheem HS, Zandi H, Majeed HJ (2021) A DFT approach on tioguanine: exploring tio-tiol tautomers, frontier molecular orbitals, IR and UV spectra, and quadrupole coupling constants. J Mol Liq 334:116018

62. Harismah K, Mirzaei M, Ghasemi N, Nejati M (2018) Non-covalent functionalisation of $\mathrm{C} 30$ fullerene by pyrrole-n-carboxylic acid $(\mathrm{n}=$ 2, 3): density functional theory studies. Z Naturforsch A 73:51-56

63. Tanjaroon C, Mills DD, Hoyos CA, Kukolich SG (2021) Calculations and analysis of 55Mn nuclear quadrupole coupling for asymmetric top acyl methyl manganese pentacarbonyl. Chem Phys Lett $762: 138151$

Publisher's Note Springer Nature remains neutral with regard to jurisdictional claims in published maps and institutional affiliations. 